\title{
Muscle activation variability is inversely correlated with walking speed
}

\author{
F. Dzeladini, A. Grappe, C. Simpson, A. R. Wu, and A. Ijspeert
}

\begin{abstract}
Individuals with motor impairments typically walk at much slower speeds than their unimpaired counterparts, yet their gait data is still evaluated against the relatively faster gait of healthy subjects. Therefore a good understanding of unimpaired gait at extremely slow speeds is needed for comparison. Studies have shown that walking at very slow speeds is quantitatively different from self-selected walking speed. These modifications can be observed at different levels (kinetic, kinematic, electromyographic). In order to better understand the changes in walking at extremely slow speeds, we recorded seven subjects walking at their preferred speed and at speeds ranging from $0.11 \mathrm{~m} / \mathrm{s}$ to $0.61 \mathrm{~m} / \mathrm{s}$. In this study, we analyzed changes in muscle activations and quantified their variability using the Pearson correlation coefficient. Confirming previous observation, we show that both the inter- and intrasubject variability of muscle activities increases with decreases in walking speed, with a more pronounced effect for proximal muscles. The inter-subject correlation of muscle activities also suggests a modular organization of muscle activities in three functional blocks at normal speed. This modular organization vanishes with decreasing walking speed following a proximodistal gradient.
\end{abstract}

\section{INTRODUCTION}

Reported preferred walking speeds for healthy adults range from $1.29 \mathrm{~m} / \mathrm{s}$ to $1.40 \mathrm{~m} / \mathrm{s}$ [1], [4], [5] while individuals with gait impairments walk at much slower speeds (e.g. SCI patients walk at speeds of 0.2 to $0.8 \mathrm{~m} / \mathrm{s}$ [5]). Very few studies have investigated whether the nervous system controls muscles differently at very slow speeds than at selfselected speeds. However, den Otter et al. demonstrated differences in muscle activity (i.e. processed electromyographic (EMG) signals) in some muscles at extremely slow speeds, possibly due to increased difficulty of maintaining postural stability at slower walking speeds [2]. Normative data of healthy human slow walking neuromechanics is necessary to better understand how to diagnose, assist, and rehabilitate individuals with motor impairments. In this paper, we contribute to the limited library of data of slow walking data in humans and examine statistical similarities between muscle activations both within and across subjects. We then use similar measures to study the modification of the modular organization of muscular activities as speed changes. We hypothesize that such compensation strategies originate from the nervous system and result in increasing variability of muscle activities with decreasing speeds.

The work performed as part of the the SYMBITRON project, supported by EU research program FP7, FET-Proactive initiative "Symbiotic humanmachine interaction" (ICT-2013-10) under project contract \#611626.

F.D., A.G., A.W., and A.I. are with the Biorobotics laboratory of the Ecole Polytechnique Federale de Lausanne, Lausanne, Switzerland. C.S. is with the mechanical engineering department at Stanford University. florin.dzeladinidepfl.ch

\section{MATERIALS AND METHODS}

\section{A. Experiment}

To test if humans use different strategies when walking at ultra-slow speeds, we asked seven healthy subjects (five females and two males, 23 to 31 years of age, body mass $M=63.8 \pm 10.1 \mathrm{~kg}$, leg length $L=0.85 \pm 0.08 \mathrm{~m}$, mean \pm s.d.) to walk on a treadmill at five different speeds $(0.11,0.33,0.5,0.61 \mathrm{~m} / \mathrm{s}$, and at their self-selected speed) for two minutes each with self-selected speeds ranging from $0.89 \mathrm{~m} / \mathrm{s}$ to $1.14 \mathrm{~m} / \mathrm{s}$. Electromyographical (EMG) data was collected from 8 muscles ( 1 - Tibialis Anterior (TA), 2 Soleus (SOL), 3 - Gastrocnemius (GAS), 4 - Vastus Medialis (VM), 5 - Vastus Lateralis (VL), 6 - Rectus Femoris (RF), 7 - Semitendinosus (ST), and 8 - Gluteus Maximus (GMAX)) of the right leg at $2 \mathrm{kHz}$ (Delsys Inc., Natick, Massachusetts, USA). To determine gait events, ground reaction forces were recorded simultaneously at $1000 \mathrm{~Hz}$ (Motekforce Link, Netherlands).

\section{B. Data Analysis}

Raw EMG signals were processed - high-pass filtered (f $=10 \mathrm{~Hz}$ ), full-wave rectified, and then low-pass filtered ( $\mathrm{f}=$ $400 \mathrm{~Hz}$ ) - to remove movement artifacts and compute muscle activation profiles. Stance and swing phases were determined based on the vertical ground reaction force with a threshold of $10 \mathrm{~N}$ (stance if the GRF exceeded the threshold and in the swing position otherwise). Swing-to-stance and stanceto-swing transitions are referred as heel contact (HC) and toe off (TO) events, respectively.

Muscles activation were time-normalized to the gait cycle, defined as right heel-strike to right heel-strike.

Outlying trials were removed via the process described in [3], i.e. if the maximum activation value of a muscle at a time point of a gait cycle exceeded the median activation plus 3 times the median absolute deviation (MAD) at this time point, the entire step was removed. Finally, muscle activations were normalized using the maximum recorded muscle activation achieved over all steps at all speeds (after the outlier removal process). Normalization of muscle activation signals was thus performed per subject and not per speed.

\section{Muscle activation variability measure}

We extracted the intra-subject and inter-subject correlations across speeds using the Pearson correlation coefficient. We define three measures of similarity :

1) Intra-subject correlation $C(m, c)_{\text {intra }}$.

For a given subject, a specific muscle $m$ and speed $c$, 
$C(m)_{\text {intra }}=\sum(\operatorname{corr}(A(m))-1) /(N-1)$ measures the similarity of muscle activations within trials, i.e it is a measure of how consistent the muscle activations are within a specific trial. $A(m)$ is a $N x T$ matrix where $N$ is the number of strides and $T$ is the number of samples per stride (here we used $T=100$ ), and $m$ specifies the muscle being considered. The Pearson correlation function, corr, outputs an $N x N$ matrix of stride similarity.

2) Inter-subject correlation $C(m)_{\text {inter }}$

This measure assesses, for a given muscle, $m$, the similarity of the averaged muscle activity between subjects at each speed and therefore measures the similarity of the averaged muscles activation between each subject. This measure is defined as: $C(m)_{\text {inter }}=$ $\mid\left(\sum \frac{\left.\operatorname{corr}\left(A_{\text {ave }}(m)\right)\right)-1}{M-1} \mid\right.$, where $A_{\text {ave }}(m)$ is a $S x T$ matrix of averaged stride muscle activation for each subject $s$. Finally, $S$ and $T$, are subject numbers and sample numbers per stride respectively.

3) Cross-muscle correlation $C(c)_{\text {cross }}$

This measure assesses - for a given speed condition $c$ - the similarity between the muscles and reveals us about the modular organization of the muscle control.
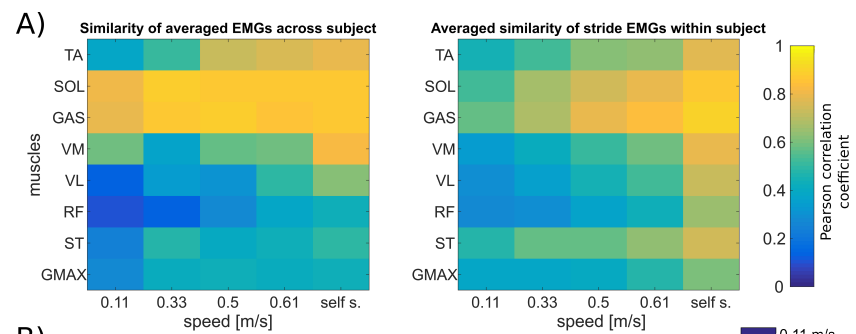

B)

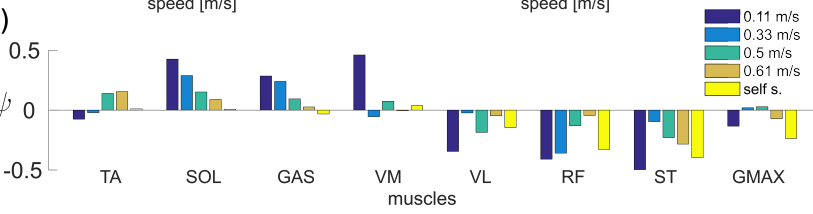

Fig. 1. A. Left: inter-subject correlation, Right: intra-subject correlation B. $\psi$ tells us if the similarity is higher for the averaged patterns across subjects or within subjects $(\psi>0$ means that the averaged EMGs patterns are more similar between the subject than the EMGs patterns within the subject, and vice-versa for $\psi<0$ ). The figures shows that muscles become less correlated at slower walking speeds. Distal muscles (SOL and GAS), are more correlated than proximal muscles (GMAX and ST).

\section{RESULTS}

Our analyses show an increase in muscle activations variability as walking speed decreases, see Fig.1 A, Left. Interestingly, the trend is more pronounced for proximal muscles than distal muscles. Fig. 1 A, Right shows the similarity of the averaged muscle activations across subjects, which also shows an increase in variability as the speed decreases. As expected, patterns across subjects are very stable across all speeds for the SOL and GAS. Interestingly, the averaged patterns of those muscles is more similar when compared between subjects than within a subject at slow speeds, meaning that muscle activations show more vari-

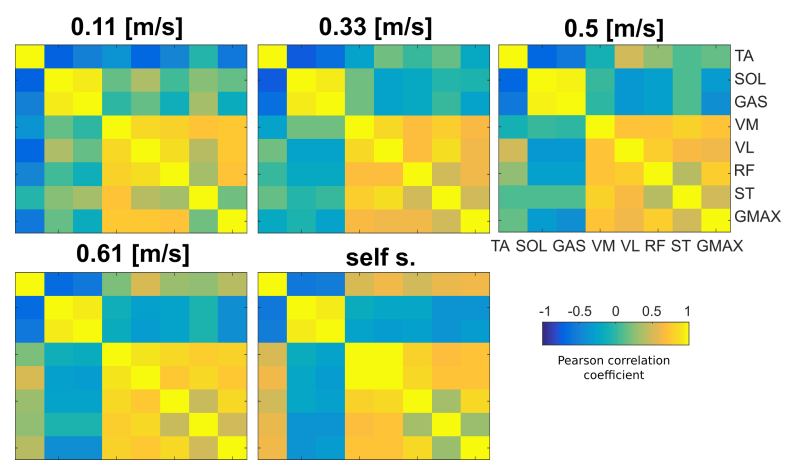

Fig. 2. Inter-subjects muscle activities. Each block shows the correlation matrices of the EMGs (averaged between subject) for the different speeds. This shows us that there is a modular organization in the data.

ability between cycles but the overall patterns are conserved within subjects, see Fig. 1 B.

The correlation analysis across muscles and subjects revealed that the muscles activation are organized in three different functional blocks (TA, SOL and GAS, hip muscles), Fig. 2. The first and third blocks showed an increase in correlation with increase in speed while the second block showed high correlation at all speeds. Interestingly, the most stable module at all speeds was the module responsible for push off (i.e. acting on plantar-flexor muscles).

\section{CONCLUSION}

Due to the possibly increased difficulty of maintaining balance at increasingly slow walking speeds [2], we see increases in muscle activation variability that could be the result of a higher degree of intervention from the nervous system (via reflex loops or higher level control). This increase in variability is correlated with decreases in speed, which suggests that we might expect that the control strategies used for self-selected walking speeds might be inadequate to model or control slower walking speeds. Therefore gait changes at very slow speeds, highlighted in this study, should be considered when developing rehabilitation strategies for motor impaired populations or designing assistive robotic coworkers or humanoid robots.

\section{REFERENCES}

[1] Raymond C Browning, Emily A Baker, Jessica A Herron, and Rodger Kram. Effects of obesity and sex on the energetic cost and preferred speed of walking. Journal of Applied Physiology, 100(2):390-398, 2006.

[2] AR Den Otter, ACH Geurts, T Mulder, and Jaak Duysens. Speed related changes in muscle activity from normal to very slow walking speeds. Gait \& posture, 19(3):270-278, 2004.

[3] Christophe Leys, Christophe Ley, Olivier Klein, Philippe Bernard, and Laurent Licata. Detecting outliers: Do not use standard deviation around the mean, use absolute deviation around the median. Journal of Experimental Social Psychology, 49(4):764-766, 2013.

[4] Betty J Mohler, William B Thompson, Sarah H Creem-Regehr, Herbert L Pick Jr, and William H Warren Jr. Visual flow influences gait transition speed and preferred walking speed. Experimental brain research, 181(2):221-228, 2007.

[5] Jennifer R Nymark, Suzanne J Balmer, Ellen H Melis, Edward D Lemaire, and Shawn Millar. Electromyographic and kinematic nondisabled gait differences at extremely slow overground and treadmill walking speeds. Journal of rehabilitation research and development, 42(4):523, 2005. 